\title{
Searching for doubly-charged vector bileptons in the Golden Channel at the LHC
}

\author{
B. Meirose ${ }^{\circledast}$ \\ Department of Physics \\ Division of Experimental High-Energy Physics \\ Sölvegatan 14, 223 62 Lund, Sweden \\ A. A. Nepomucend円 \\ INFES \\ Universidade Federal Fluminense \\ Cx.Postal 68528, Santo Antonio de Padua, RJ, Brazil
}

(Dated: July 9, 2018)

\begin{abstract}
In this paper we investigate the LHC potential for discovering doubly-charged vector bileptons considering the measurable process $p, p \rightarrow e^{\mp} e^{\mp} \mu^{ \pm} \mu^{ \pm} X$. We perform the study using four different bilepton masses and three different exotics quark masses. Minimal LHC integrated luminosities needed for discovering and for setting limits on bilepton masses are obtained for both $7 \mathrm{TeV}$ and $14 \mathrm{TeV}$ center-of-mass energies. We find that these spectacular signatures can be observed at the $\mathrm{LHC}$ in the next years up to a bilepton mass of order of $1 \mathrm{TeV}$.
\end{abstract}

PACS numbers: $12.60 . \mathrm{Cn}, 14.70 . \mathrm{Pw}$

\section{INTRODUCTION:}

Although bileptons [1] can be considered as exotic particles relative to their Standard Model (SM) cousins due to their unfamiliar quantum numbers, they are, in a sense, a conservative prediction. Even though they are suggested only by special extensions of the SM, their existence is, in our view, no more special than other proposals such as weak-scale extra dimensions or supersymmetry (SUSY). Indeed bileptons are a prediction employing only the model building rules for renormalizable gauge theories so successful in the Standard Model. The main motivation for expecting bileptons is that they explain three quark-lepton families.

Generically speaking, a bilepton is a boson which couple to two leptons, but not to SM quarks, and which carries two units of lepton number. They are present in several beyond-SM scenarios, such as left-right symmetric models, technicolor and theories of grand unification. The bileptons in which we are interested are doubly charged vector bosons which couple to SM leptons, and are predicted when the Standard Model is embedded in a larger gauge group. The so-called 331 models [2, 3] fall into this category and in this article we restrict ourselves to this case. However, as will be explained in section II, we expect our results to hold in any model containing vector bileptons.

In bilepton pair production, each of the bileptons will decay to two same-sign leptons. Therefore, they provide an exceptionally clean signature of four isolated high transverse momentum leptons, not necessarily of the

\footnotetext{
*Electronic address: Bernhard.Meirose@cern.ch
}

${ }^{\dagger}$ Electronic address: Andre.Asevedo@cern.ch same flavor. In this article we explore some of the consequences of this fact at the LHC. We study the actual collider signatures for the process $p, p \rightarrow e^{\mp} e^{\mp} \mu^{ \pm} \mu^{ \pm} X$ which has no SM background and is for this reason a "golden" channel for finding bileptons. In this process, a bilepton pair is produced via $s$ or $t$ channel where one of them decays into electrons while the other decays into muons.

The most useful current lower bound on vector bileptons require these particles to be heavier than $740 \mathrm{GeV}$ [4]. This limit has been derived from experimental limits on fermion pair production at LEP and lepton-flavor charged lepton decays. Another useful lower bound is $M_{Y}>850 \mathrm{GeV}$, a result which was established from muonium-antimuonium conversion [5]. Although more stringent, this limit depends on the assumption that the bilepton coupling is flavor-diagonal. In this article we nevertheless consider bilepton masses as low as $400 \mathrm{GeV}$, following a similar line of reasoning as [6], where the authors argued a lower bound of $350 \mathrm{GeV}$ for doublycharged vector bilepton masses, a limit that is compatible with other low energy bounds 7]. We also allow a larger upper bound for bilepton masses in 331 models than the usual $1 \mathrm{TeV}$ considered by some authors.

This article has been organized as follows. In section II we present our motivations to perform the present study. In section III we explain the numerical procedure for simulating the $p, p \rightarrow e^{\mp} e^{\mp} \mu^{ \pm} \mu^{ \pm} X$ reaction as well as its validation. In section IV we show relevant experimental observables for the bilepton golden channel. In section $\mathrm{V}$ we present the discovery potential for bileptons, calculating mass exclusion limits as a function of the LHC integrated luminosities, including a digression on the prospects for the accelerator's $7 \mathrm{TeV}$ run and for the super LHC (sLHC). We conclude in section VI. 


\section{MOTIVATIONS}

Many interesting channels have been studied in the literature concerning bileptons, but curiously, there has been no systematic study on the phenomenology of the bilepton golden channel at the LHC. The authors in [8] did a fairly comprehensive study of bilepton phenomenology at hadron colliders. But in all cases they limited themselves to bilepton pair-production study, disregarding its decays. In the present study we go a step further in understanding the actual collider signatures, by considering measurable final states.

\section{A. 331 models}

The 331 models are based on the gauge symmetry $S U(3)_{C} \otimes S U(3)_{L} \otimes U(1)_{X}$, hence their name. Their first versions appeared in 1992 [2, 3]. There are many interesting aspects of the 331 models worth noticing but the most intriguing one is the explanation of three quarklepton families, which is the main motivation for expecting bileptons in Nature. This is done via a nontrivial anomaly cancellation in the 331 Model that takes place between families, which is achieved by requiring the number of families to be equal to the number of quark colors. The explanation of the number of generations is also arguably one of the main reasons that keep model builders interested in 331 models, since they are one of the few which elegantly address this problem. The 331 models are also the simplest extension of the SM containing bileptons. Other interesting features of the 331 models include: a) they treat the third generation differently than the first two, this lead to an explanation of the heavy top quark mass; b) they have an automatic Peccei-Quinn symmetry [9], hence they are also able to solve the strong $\mathrm{CP}$ problem. Moreover, gauge symmetry $S U(3)_{C} \otimes S U(3)_{L} \otimes U(1)_{X}$ is considered a subgroup of the popular $E_{6}$ [10] Grand Unified Theory (GUT), which can be itself derived from $E_{8} \otimes E_{8}$ [11] heterotic string theory. Finally, it is worth mentioning that contrary to the SM, in 331 models lepton family number is not required to be conserved, only total lepton number. There is already experimental proof that lepton family number is not an exact symmetry via neutrino oscillations and one can regard this as circumstantial evidence for the non conservation of lepton family number in general. The combination of such intriguing aspects make bileptons desirable candidates to be found in Nature.

Another important point to be discussed is the minimal version of the 331 models. There are different ways in which $S U(3)_{C} \otimes S U(3)_{L} \otimes U(1)_{X}$ can be broken down back to the $S U(3)_{C} \otimes S U(2)_{L} \otimes U(1)_{Y}$ SM gauge symmetry. The minimal version corresponds to use minimal Higgs structure to achieve this goal. In this version it is required that the new neutral vector boson $Z^{\prime}$ mass term to be coupled to the bilepton mass, like:

$$
\frac{M_{Y}}{M_{Z^{\prime}}}=\frac{\sqrt{3\left(1-4 \sin ^{2} \theta_{W}\right)}}{2 \cos \theta_{W}}
$$

Regarding theoretical upper bounds in 331 models there is no consensus in the literature. It was reasoned in [3] that in 331 models, bileptons cannot be significantly heavier than $1 \mathrm{TeV}$, because of an upper limit in the symmetry breaking scale which is placed by requiring the sine squared of the Weinberg mixing angle $\left(\theta_{W}\right)$ to be smaller than $1 / 4$, which is the same line of argumentation used in [12] to conclude that the $Z^{\prime}$ mass cannot be itself heavier than $3.1 \mathrm{TeV}$. Considering the mass relation between $Z^{\prime}$ and the bilepton, it could be argued that at least the minimal version of the 331 model could be excluded, should bileptons not be detected at the LHC, since any vector bilepton mass heavier than $\sim 840 \mathrm{GeV}$ would violate the $Z^{\prime}$ mass upper limit via the mass relation between the two gauge bosons given by equation (1). This conclusion was challenged in [13]. The argument is as follows. The 331 model predicts that there is an energy scale $\mu$ where the model loses its perturbative character. Should experimental data suggest a lower bound on the vector bilepton mass larger than $\mu$, the model would be ruled out. The value of $\mu$ is calculated through the condition $\sin ^{2} \theta_{W}(\mu)=1 / 4$, but from this requirement alone it is not possible to know the real value of $\mu$. Then the upper limit on the vector bilepton mass could be, for instance, $3.5 \mathrm{TeV}$, as has been discussed by [14]. By the same token the $3.1 \mathrm{TeV}$ upper limit in the $Z^{\prime}$ mass is automatically challenged. Therefore we do not believe it is possible to unambiguously discard any 331 model at the LHC (although they could be discovered at it), since we consider the bilepton mass upper bound to reasonably lie beyond the accelerator's reach.

For the lower bounds on vector bileptons we consider at least two mass points that violate the general $740 \mathrm{GeV}$ limit imposed by LEP data. As explained by the authors in [6], all the constraints on the 331 parameters coming from experiments involving leptonic interaction should be examined with caution. In the 331 model the leptons mix by a Cabibbo-Kobayashi-Maskawa-like mixing matrix whose elements have not yet been measured, so usually these experiments (and derived limits) apply only when the leptonic mixing matrix is diagonal. Also, in models with an extended Higgs sector some not unrealistic situations could exist in which the scalar bosons contribution to muonium to antimuonium conversion is not negligible. This puts also the possibility of strengthening bilepton experimental limits still at the LHC's 7 $\mathrm{TeV}$ run in a new perspective, a possibility that we also discuss in this article.

\section{B. 331 Models and Supersymmetry}

In recent years, a considerable fraction of both the experimental and theoretical communities has dedicated it- 
self to supersymmetry. It is doubtlessly the mainstream subject in particle physics. The 331 models are not necessarily supersymmetric. But any renormalizable gauge theory can be extended to a globally supersymmetric model. The 331 models, being anomaly free, are renormalizable and fall of course in this category. Some authors have explored this possibility 15]. Furthermore, as it was argued also in [13], in this model, the hierarchy problem is less severe than in the SM and its extensions since no arbitrary mass scale can be introduced. The masses of fundamental scalars are sensitive to the mass of the heaviest particles which couple directly or indirectly with them. Since in the 331 model the heaviest mass scale is of the order of a few TeVs there is not a hierarchy problem at all. This feature remains valid when supersymmetry is introduced. Thus, the breaking of the supersymmetry is also naturally at the $\mathrm{TeV}$ scale in the 331 model.

\section{Model independent vector bilepton searches at the LHC}

Ideally one would like to study bileptons in hadron colliders as model independent as possible. For vector bileptons this is not possible as the non-inclusion of the $Z^{\prime}$ boson makes bilepton pair production to violate unitarity, in complete analogy to what happens with $e^{+} e^{-} \rightarrow W^{+} W^{-}$using only photon exchange. This is another motivation to use the 331 model, although we do not restrain ourselves to the minimal version, allowing $M_{Y}$ and $M_{Z^{\prime}}$ to vary independently of one another. Even though model-dependent, our cross-sections should approximately be in the same order of magnitude with any other model containing bileptons, since at hadron colliders these particles have to be produced by the same Drell-Yan pair production process, regardless of the model. Exotic heavy quark exchange can influence this scenario, a possibility we do explore and which make our conclusions even more general.

\section{NUMERICAL IMPLEMENTATION AND VALIDATION}

To simulate the bilepton golden channel at the LHC we have implemented the 331 model in the Comphep generator [16]. We followed reference [17] to implement the bilepton trilinear gauge interactions and 8] for the $Z^{\prime}$ couplings with fermions. For the bilepton interaction with leptons we have used the Lagrangian expression given in [18], generating the respective couplings using the Lanhep package [19]. We also take into account bilepton interactions with exotic quarks in the 331 model. For this, we considered the following interactions with the exotic quark sector:

$$
\mathcal{L}_{Q}=-\frac{g}{2 \sqrt{2}}\left[\bar{Q}^{c} \gamma^{\mu}\left(1-\gamma_{5}\right) q Y_{\mu}^{++}\right]+\text {H. c. },
$$

where $\mathrm{Q}\left(D_{1}, D_{2}, T_{1}\right)$ is an exotic heavy quark in the 331 model, $\mathrm{q}$ is a SM quark and $g=e / \sin \theta_{W}$ as in the $\mathrm{SM}$. We considered the respective interaction pairs for $(Q, q)$ to be $\left(D_{1}, u\right),\left(D_{2}, c\right)$ and $\left(T_{1}, b\right)$, where $u, c$ and $b$ are SM quarks. Our purpose in including such interactions was, besides studying the influence of the heavy quark sector, to guarantee that all relevant quark subprocesses $q \bar{q} \rightarrow Y^{++} Y^{--}$respect unitarity. Since the 331 model itself does not determine the elements of the mixing matrix (which determines how bileptons interact with exotic quarks), this is a reasonable criteria. This was needed for $u \bar{u} \rightarrow Y^{++} Y^{--}, b \bar{b} \rightarrow Y^{++} Y^{--}$and $c \bar{c} \rightarrow Y^{++} Y^{--}$which violate unitarity otherwise. In Figure 1 , one can see the $u \bar{u} \rightarrow Y^{++} Y^{--}$reaction crosssection as a function of the center of mass energy. Note that up to energies beyond the LHC designed centerof-mass-energy of $14 \mathrm{TeV}$, the cross-section dependence with energy behaves as expected. We tested this fact for all quarks involved in the proton parton distribution function (PDF), CTEQ611 [20] that was used for the complete $p, p \rightarrow e^{\mp} e^{\mp} \mu^{ \pm} \mu^{ \pm} X$ reaction simulation.

Concerning particle parameters, we considered heavy quark masses to be equal to $400 \mathrm{GeV}, 600 \mathrm{GeV}$ and 800 $\mathrm{GeV}$ (lower bounds on exotic supersymmetric particles impose a lower bound on 331 exotic quark masses to be $\sim 250 \mathrm{GeV}$ [21]) and used $1 \mathrm{TeV}$ for the $Z^{\prime}$ mass, since $331 Z^{\prime}$ masses below $920 \mathrm{GeV}$ were excluded using results from the CDF collaboration [22]. For the doubly charged bileptons $Y^{++}$we considered four mass points: 400, 600, $800, \mathrm{GeV}$ and $1 \mathrm{TeV}$. For each mass point, $10000 p, p \rightarrow$ $e^{\mp} e^{\mp} \mu^{ \pm} \mu^{ \pm} X$ events were generated with Comphep.

The $Y^{++}, Z^{\prime}$ and exotic quarks widths were calculated directly in Comphep for each mass point.The $Z^{\prime}$ width for $M_{Q}=400 \mathrm{GeV}$ is $\Gamma_{Z^{\prime}} \sim 360 \mathrm{GeV}$. For the other two exotics quark masses, $\Gamma_{Z^{\prime}}$ drops to $\sim 155 \mathrm{GeV}$, since Z' decay into exotic quarks becomes kinematically forbidden. The variation of $\Gamma_{Z^{\prime}}$ with respect to $M_{Y}$ is of order of $1 \%$ between the highest and lowest bilepton mass considered.

To further cross-check our implementation we reproduced the results from reference [8] Figure 4, for bilepton pair production at the LHC using $M_{Z^{\prime}}=1 \mathrm{TeV}$. Minor numerical differences in the cross-sections can easily be explained by the use of different PDF's.

\section{OBSERVABLES}

In what follows, all histograms were produced considering an integrated luminosity of $100 \mathrm{fb}^{-1}$ and the nominal LHC energy of $14 \mathrm{TeV}$, unless otherwise stated. 


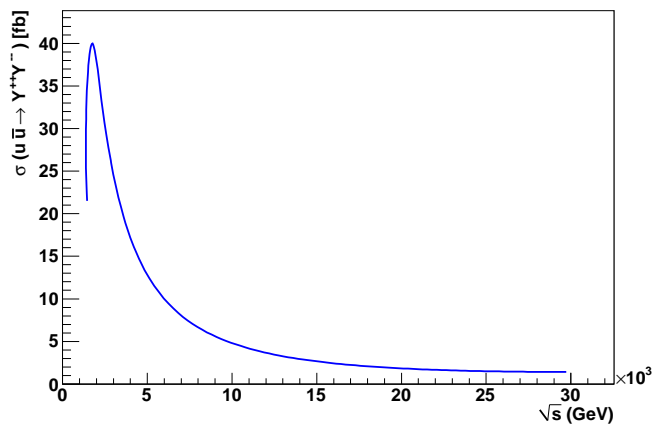

FIG. 1: $u \bar{u} \rightarrow Y^{++} Y^{--}$cross section as a function of $\sqrt{s}$. The subprocess correctly respects unitarity.



FIG. 2: Total cross-section of the process $p, p \rightarrow e^{\mp} e^{\mp} \mu^{ \pm} \mu^{ \pm} X$ as a function of bilepton mass. The black/solid, blue/dotted and red/dashed lines represent the cross-section for $M_{Q}=$ 400, $M_{Q}=600$ and $M_{Q}=800 \mathrm{GeV}$, respectively.

\section{A. Cross section and Width}

Figure 2 shows the total cross-section of the $p, p \rightarrow$ $e^{\mp} e^{\mp} \mu^{ \pm} \mu^{ \pm} X$ process as a function of the doubly-charged bilepton mass, for three different exotic quark masses. Here one can see clear evidence on the problem of the influence of the heavy quark sector on bilepton production.

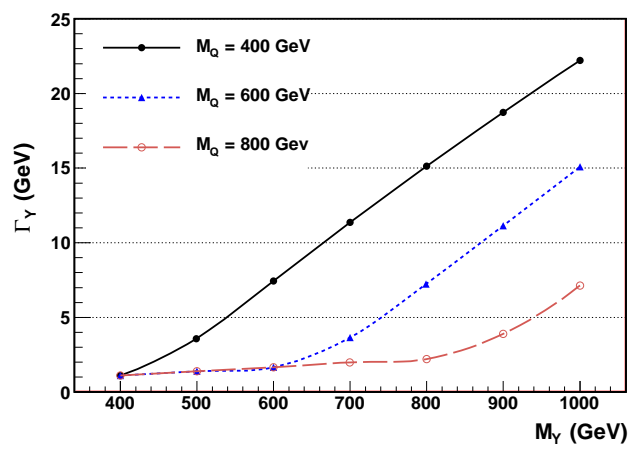

FIG. 3: Bilepton width as a function of bilepton mass for three different quark mass values.
Note that for a bilepton of $M_{Y}=800 \mathrm{GeV}$ the effect on the cross-section on having heavier exotic quark masses of $M_{Q}=800 \mathrm{GeV}$ as compared to $M_{Q}=400 \mathrm{GeV}$ is to increase the cross-section of the the process by a factor of $\sim 30$. We can also see that the $M_{Q}=600$ and $M_{Q}$ $=800$ curves split at $M_{Y}=600 \mathrm{GeV}$. This happens because when $M_{Y}>M_{Q}$, bilepton decays like $Y^{ \pm \pm} \rightarrow q Q$ becomes kinematically allowed, which makes the value of $\operatorname{Br}\left(Y^{ \pm \pm} \rightarrow l^{ \pm} l^{ \pm}\right)$decrease for a given bilepton mass.

Figure 3 shows the bilepton width as a function of bilepton mass for the same exotic quark masses as before. Here we can see how the width increases when new decays are allowed. It is also clear from the plot that the bilepton resonance is very narrow.

We admittedly used a very simple approach to the problem of how bileptons and heavy quarks actually mix. However, different ways on determining the values of the mixing matrix between bileptons and heavy quarks could, in principle, intensify such effects even more drastically. An open problem that would deserve a separate study of its own.

\section{B. Pseudorapidity}

In order to investigate a more realistic scenario, we require the events that have been generated to pass some selection criteria according to the LHC detectors. First, the four leptons must be within the detectors geometrical acceptance, i.e., $|\eta|<2.5$ [23]. With this requirement, the fraction of selected events goes from $83 \%$ for $M_{Y}$ $=400 \mathrm{GeV}$ to $93 \%$ for $M_{Y}>900 \mathrm{GeV}$. Additionally, we also require each lepton $p_{T}$ be greater than $20 \mathrm{GeV}$. The loss of efficiency due this cut is negligible. Finally, we assume a reconstruction efficiency of $60 \%$. Figure 4 illustrates the electron pair pseudorapidity distribution of the events before and after the selection for $M_{Y}=600$ $\mathrm{GeV}$ where we can see the fraction of surviving events. The acceptance (geometrical acceptance $\times$ efficiency) for this case is $53 \%$, and this changes by $\pm 3 \%$ depending on the bilepton mass. The pseudorapidity distribution for muons is similar.

\section{Transverse Momentum}

In Figures 5 and 6 it is respectively shown the transverse momentum distribution for both the final state selected electrons and muons pairs of the golden channel, where both the doubly-charged bilepton and the heavy quarks have a mass of $600 \mathrm{GeV}$. Typically most of the events are produced in a region between $\sim 200 \mathrm{GeV}$ and $400 \mathrm{GeV}$ but the tail of the distribution has few events going up to $900 \mathrm{GeV}$ for the electron pairs. 


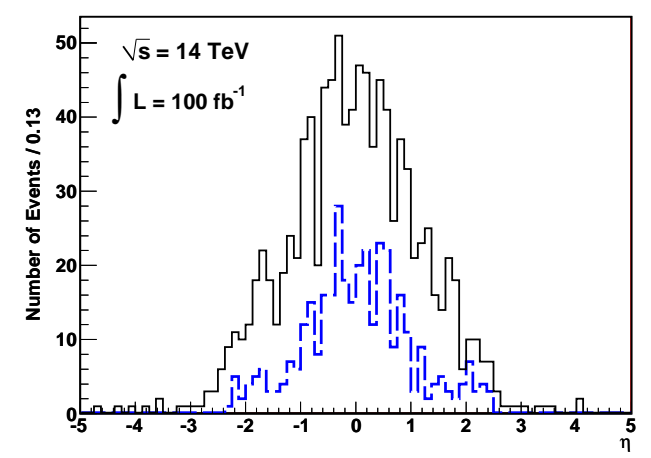

FIG. 4: Electron pair pseudorapidity distribution for $M_{Y}$ $=M_{Q}=600 \mathrm{GeV}$ before (black/solid line) and after (blue/dashed line) selection .

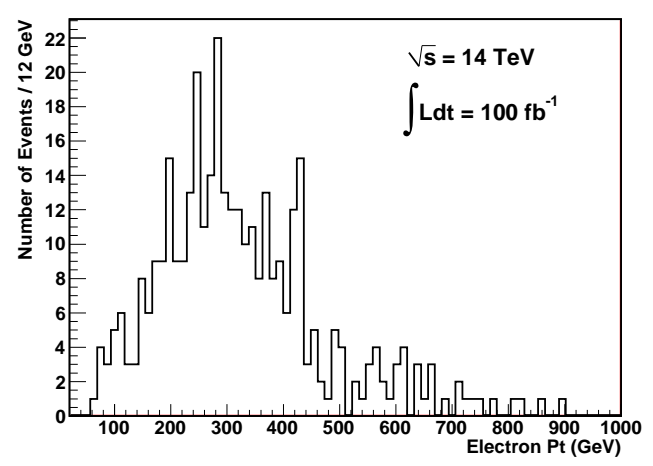

FIG. 5: Electron pair transverse momentum distribution for $M_{Y}=M_{Q}=600 \mathrm{GeV}$.

\section{Invariant Mass}

When doubly-charged vector bileptons are produced in pairs each bilepton can decay to a pair of same-sign leptons, not necessarily of the same flavor, where each lepton pair will have the same invariant mass distribu-

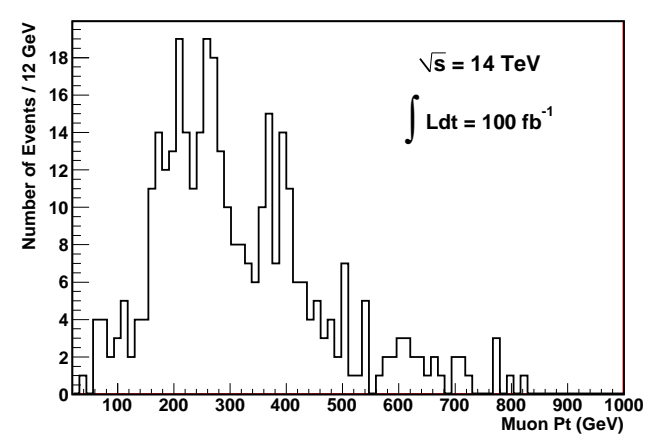

FIG. 6: Muon pair transverse momentum distribution for $M_{Y}$ $=M_{Q}=600 \mathrm{GeV}$.

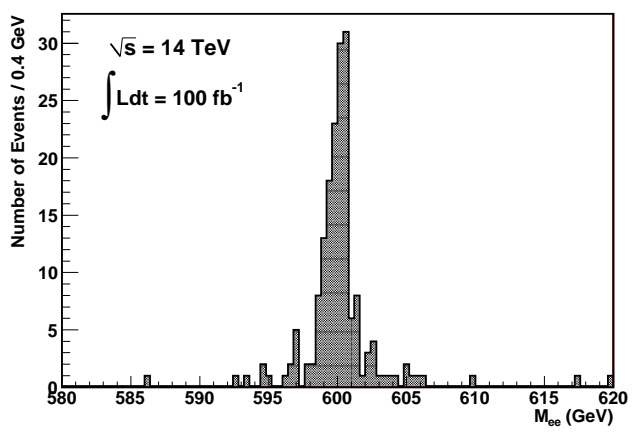

FIG. 7: Invariant mass for electron pair, for a bilepton of 600 $\mathrm{GeV}$.

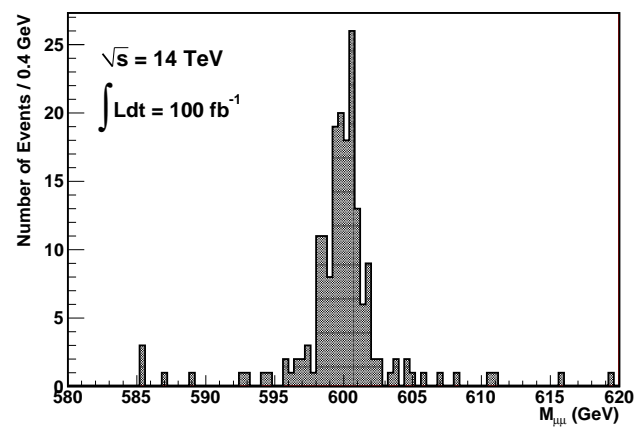

FIG. 8: Invariant mass for muon pair, for a bilepton of 600 $\mathrm{GeV}$.

tion. This would be the most compelling evidence of a new resonance coming from a bilepton and very strong evidence of new physics. This is displayed in Figures 7 and 8 for the final electron and muon pairs respectively, considering both the bilepton and exotic quarks masses to be equal $600 \mathrm{GeV}$. The mean of the invariant mass for both the electrons and muon pairs in the final state unmistakably peaks at $600 \mathrm{GeV}$, the bilepton mass. Such a plot will of course demand several years of data taking such that enough statistics can be gathered, especially for higher bilepton masses, as we will address in the next section.

\section{DISCOVERY POTENTIAL AND LIMITS}

In order to determine the LHC potential to find bileptons, we calculate the minimal LHC integrated luminosity needed for a five-sigma bilepton discovery. For each bilepton mass, the detector acceptance as stated in section IV.B is considered and the $5 \sigma$ significance is obtained by requiring 5 events with two electrons and two muons in the final state to be produced. The minimal integrated luminosity $L_{\text {int }}$ is given by 


$$
L_{i n t}=\frac{5}{\varepsilon\left(M_{Y}\right) \sigma\left(M_{Y}\right)}
$$

where $\varepsilon\left(M_{Y}\right)$ is the detector acceptance and $\sigma\left(M_{Y}\right)$ is the cross seciton. Figure 9 shows the calculated values of $L_{i n t}$ as a function of bilepton mass. From the plot, we conclude that a integrated luminosity of order of 10 $\mathrm{pb}^{-1}$ is enough for discovering a bilepton of $400 \mathrm{GeV}$ mass, which means that such signal can be observed at the very early days of LHC running with $14 \mathrm{TeV}$, even in a regime of low luminosity. Depending on the exotic quark mass, luminosities of order $10 \mathrm{fb}^{-1}$ to $100 \mathrm{fb}^{-1}$ are needed to discover bileptons if $M_{Y}=800 \mathrm{GeV}$. These scenarios can be achieved after 1 year of LHC operation in low and high luminosity regimes, respectively. Finally, for $M_{Y}=1 \mathrm{TeV}$, around 10 years of LHC operation at high luminosity would be needed for the resonance observation. This is in contrast with what would happen at the ILC where Møller and Bhabha scattering receive both huge corrections from virtual vector bileptons [24], so that the bilepton mass reach can be as high as $\sim 11$ $\mathrm{TeV}$, provided polarized beams are used.

Figure 10 shows the integrated luminosity required for excluding bileptons at 95\% CL as function of the bilepton mass. For this estimation, we have used the D0 limit calculator 25] to set upper limits on the cross sections that are consistent with the observation of zero events in data, assuming no background. The Bayesian technique is used to set this limits. In this approach, given a posterior probability density function for the signal crosssection, the upper limit on the signal cross section $\sigma_{\text {up }}$, specified at some confidence level $100 \times \beta \%$, is given by

$$
\beta=\int_{0}^{\sigma_{\mathrm{up}}} d \sigma \rho(\sigma \mid k, I)
$$

where $\beta=0.95, \rho(\sigma \mid k, I)$ is the posterior probability density function, $k$ is the number of events observed and $I$ represents prior information available.

The acceptances values used in this calculation are the same as before. We have assumed an uncertainty of $5 \%$ on the acceptance and $10 \%$ on the integrated luminosity. It also is assumed that the errors on the acceptance and luminosity are uncorrelated. The obtained limits on the cross section are then translated to limits on the bilepton mass. Comparing Figure 10 with the discovery plot, we can see that around $60 \%$ of the discovery integrated luminosity is needed for excluding bileptons of a given mass.

If a significant signal is observed, the next natural step is to determine the properties of the new particle. In order to check how well the bilepton mass can be reconstructed with the amount of data needed for discovering, we perform a unbinned maximum likelihood fit to the $M_{e e}$ and $M_{\mu \mu}$ distributions for $M_{Y}=600 \mathrm{GeV}$ and $M_{Y}$ $=800 \mathrm{GeV}$ MC samples, with a fixed exotic quark mass of $600 \mathrm{GeV}$. The probability density function used in the
TABLE I: Mean and standard deviation of fitted mass peak for dielectron and dimuon invariant mass distributions.

\begin{tabular}{|c|c|c|c|c|}
\hline & \multicolumn{2}{|c|}{$M_{Y}=600 \mathrm{GeV}$} & \multicolumn{2}{c|}{$M_{Y}=800 \mathrm{GeV}$} \\
\hline channel & $\bar{m}$ & $\sigma_{m}$ & $\bar{m}$ & $\sigma_{m}$ \\
\hline$e e$ & 599.9 & 0.5 & 799.9 & 2.4 \\
\hline$\mu \mu$ & 600.1 & 0.6 & 799.9 & 2.6 \\
\hline
\end{tabular}

TABLE II: Exclusion limits for doubly-charged bileptons with respect to LHC's integrated luminosity and 331 exotic quark masses at the $7 \mathrm{TeV}$ run. Masses are in GeV.

\begin{tabular}{|c|c|c|c|}
\hline & \multicolumn{3}{|c|}{ Integrated luminosity } \\
\hline$M_{Q}$ & $1 \mathrm{fb}^{-1}$ & $5 \mathrm{fb}^{-1}$ & $10 \mathrm{fb}^{-1}$ \\
\hline 400 & 427 & 466 & 483 \\
\hline 600 & 478 & 534 & 566 \\
\hline 800 & 478 & 534 & 566 \\
\hline
\end{tabular}

fits is a Breit-Wigner and two parameters are fitted: the position of the invariant mass peak $m$ and the resonance width $\Gamma$. For each bilepton mass, fits are performed to $1000 \mathrm{MC}$ experiments (i.e, $1000 M_{e e}$ and $M_{\mu \mu}$ distributions) and the mean values of the fitted parameters are reported. The number of events in each $\mathrm{MC}$ experiment is fixed to 5 . Table I shows the mean, $\bar{m}$, of the fitted mass values and the standard deviation of the $m$ distribution. For both bilepton masses, we see that there is a very good agreement between fitted and true masses in both channels. As expected, the spread of the distribution is larger for $M_{Y}=800 \mathrm{GeV}$. The bilepton width can also be obtained from the fit at generator level, but it will be dominated by the detector resolution in a more realistic scenario, since bileptons are very narrow resonances.

\section{LHC $7 \mathrm{TeV}$ run potential}

Considering the LHC's goals until the end of 2012 for a center-of-mass energy of $7 \mathrm{TeV}$, we estimate the potential for discovering or for setting limits on bilepton masses and couplings at this data taking stage. We consider three scenarios with 1,5 and $10 \mathrm{fb}^{-1}$ [26] of integrated luminosity. Using the D0 limit calculator and using the same values for acceptance and uncertainties as in the previous sections, we obtain the respective bilepton masses consistent with the observation of zero events for three exotic quark masses: 427, 466 and $483 \mathrm{GeV}$ for $M_{Q}=400 \mathrm{GeV}$; 478,534 and $566 \mathrm{GeV}$ for both $M_{Q}=600 \mathrm{GeV}$ and $M_{Q}=800 \mathrm{GeV}$, from the lowest to the highest integrated luminosity, respectively. These are the doubly-charged vector bilepton masses that can be excluded at 95\% CL. The $7 \mathrm{TeV}$ exclusion limits are summarized in Table II.

The $5 \sigma$ discovery potentials at 5 and $10 \mathrm{fb}^{-1}$ of integrated luminosity are respectively found to be 452,535 $\mathrm{GeV}$ for $M_{Q}=400 \mathrm{GeV}, 511,542 \mathrm{GeV}$ for $M_{Q}=600$ $\mathrm{GeV}$ and $515,544 \mathrm{GeV}$ for $M_{Q}=800 \mathrm{GeV}$. With $1 \mathrm{fb}^{-1}$ 
TABLE III: Discovery mass reach for doubly-charged bileptons with respect to LHC's integrated luminosity and 331 exotic quark masses at the $7 \mathrm{TeV}$ run. Masses are in $\mathrm{GeV}$.

\begin{tabular}{|c|c|c|c|}
\hline & \multicolumn{3}{|c|}{ Integrated luminosity } \\
\hline$M_{Q}$ & $1 \mathrm{fb}^{-1}$ & $5 \mathrm{fb}^{-1}$ & $10 \mathrm{fb}^{-1}$ \\
\hline 400 & 415 & 454 & 535 \\
\hline 600 & 459 & 511 & 542 \\
\hline 800 & 459 & 515 & 544 \\
\hline
\end{tabular}

TABLE IV: Discovery mass reach and exclusion limits for doubly-charged bileptons with respect to sLHC and 331 exotic quark masses. Masses are in $\mathrm{GeV}$.

\begin{tabular}{|c|c|c|}
\hline$M_{Q}$ & Discovery & Exclusion \\
\hline 400 & 1100 & 1170 \\
\hline 600 & 1150 & 1220 \\
\hline 800 & 1230 & 1300 \\
\hline
\end{tabular}

the reach is $459 \mathrm{GeV}$ using the highest exotic quark mass. This mass reach is way above the minimum bound of 350 $\mathrm{GeV}$, so such a discovery at this phase, is not completely discarded. In any case, even if no discoveries are made at the $7 \mathrm{TeV}$ run, the exclusion limits that will be established are still valuable for setting up the scenario for the $14 \mathrm{TeV}$ run. The $7 \mathrm{TeV}$ discovery reach results are summarized in Table III.

\section{sLHC}

The upgrade of the LHC machine, also referred as the sLHC [27] project aims at increasing the peak luminosity by a factor of 10 and deliver approximately $3000 \mathrm{fb}^{-1}$ to the experiments. Although it's rather difficult to foresee what would be interesting to study at the sLHC without having the LHC run at its nominal luminosity first, here we assume that no vector bilepton signals were found at the LHC and explore the sLHC exclusion and discovery potentials for the exotic particles. Using the same techniques described in the previous sections we find the exclusion potential for three heavy quark masses: 1170 $\mathrm{GeV}$ for $M_{Q}=400 \mathrm{GeV}, 1220 \mathrm{GeV}$ for $M_{Q}=600 \mathrm{GeV}$ and $1300 \mathrm{GeV}$ for $M_{Q}=800 \mathrm{GeV}$. The discovery potential is also increased: $1100 \mathrm{GeV}$ for $M_{Q}=400 \mathrm{GeV}, 1150$ $\mathrm{GeV}$ for $M_{Q}=600 \mathrm{GeV}$ and $1230 \mathrm{GeV}$ for $M_{Q}=800$ $\mathrm{GeV}$. This represents a gain of $\sim 200 \mathrm{GeV}$ in terms of discovery mass reach compared to the default luminosity $14 \mathrm{TeV}$ LHC run. This region is certainly worth exploring since it is still considerably below the upper limit of $3.5 \mathrm{TeV}$ we have discussed in section II. The results for the sLHC are displayed in Table IV.

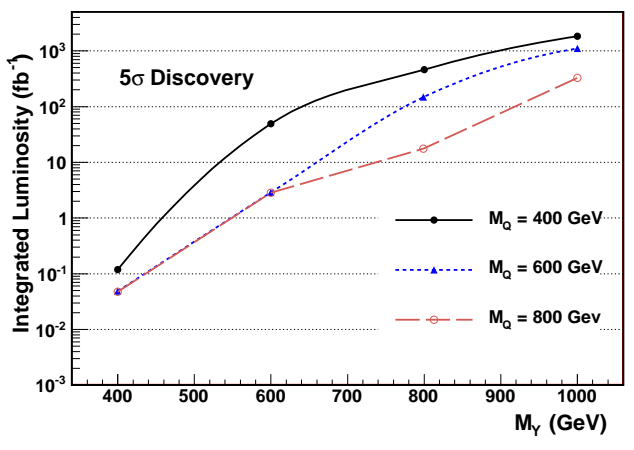

FIG. 9: Minimal integrated luminosity needed for a $5 \sigma$ bilepton discovery at the LHC.

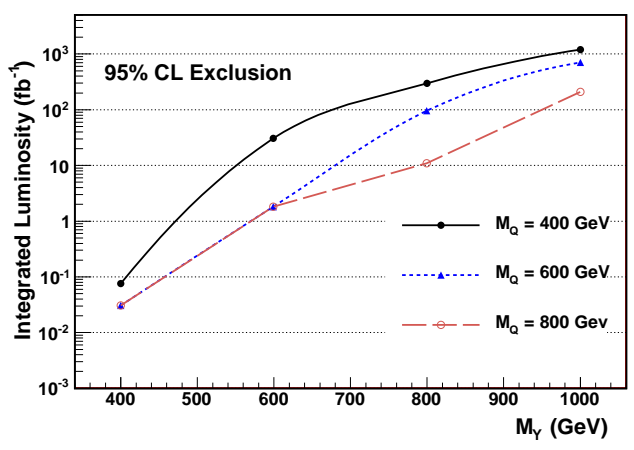

FIG. 10: Integrated luminosity needed for 95\% CL bileptons exclusion taking into account acceptance and luminosity uncertainties.

\section{CONCLUSIONS}

We have investigated the LHC potential for discovering or setting limits on doubly-charged vector bileptons, in different scenarios considering important experimental aspects in the simulation like detector geometrical acceptance, luminosity uncertainty and lepton efficiency. By analysing the observable final state $e^{\mp} e^{\mp} \mu^{ \pm} \mu^{ \pm}$, we have found that bilepton signatures can already be observed at very early stages of LHC running with $14 \mathrm{TeV}$, if the bilepton mass is not much greater than $400 \mathrm{GeV}$. On the other hand, if the bilepton mass lies in the $\mathrm{TeV}$ scale, at least 10 years of the machine operation will be needed for discovering it, if $M_{Q}>600 \mathrm{GeV}$. The observation of such signal, in combination with $Z_{331}^{\prime}$ searches in dilepton channel, would provide a very powerful way of discriminating between 331 models and other BSM scenarios which also predict heavy neutral gauge bosons. If no signal is observed, the LHC can extend considerably the currents limits on bilepton mass by direct search in the four lepton final state.

At the current LHC energy, $7 \mathrm{TeV}$, masses up to 566 $\mathrm{GeV}$ can be excluded and if $10 \mathrm{fb}^{-1}$ of data is recorded, vector bilepton masses up to $544 \mathrm{GeV}$ could be discovered. We also found that the sLHC can expand the lower 
exclusion limits up to a mass of $1300 \mathrm{GeV}$.

We also made a revision on current experimental bounds on bileptons in 331 models and the possibility of results from the LHC to exclude some versions of these models. We found that it is not possible to safely discard any 331 model, including its minimal version, at the LHC. Purely theoretical arguments taken from the literature are used to draw this conclusion.

Furthermore, we investigated how the heavy quark sector of the 331 model influences our results. In some cases a substantial change in the process cross-section is observed by varying the value of the heavy quark masses. Since some of the best previous limits on bileptons were coming from experiments containing at least one leptonic beam, we conclude that new results from the $\mathrm{LHC}$ will be indispensable in determining to a more accurate extent which models like 331 can be disfavored or discovered. The final state studied in this article will be the best channel to experimentally determine this.
[1] F. Cuypers and S. Davidson, Eur. Phy. J. C 2,503 (1998) hep-ph/9609487 and references therein.

[2] F. Pisano and V. Pleitez, Phys. Rev. D 46, 410 (1992);

[3] P. H. Frampton, Phys. Rev. Lett. 69, 2889 (1992).

[4] M. B. Tully and G. C. Joshi, Phys. Lett. B466, 333 (1993); hep-ph/9905552

[5] Willmann et al, Phys. Rev. Lett. 82, 49 (1999).

[6] Y. A. Coutinho, P. P. Queiróz Filho, M. D. Tonasse, Phys. Rev. D 60, 115001 (1999).

[7] P. H. Frampton, J. T. Liu, B. C. Rasco, and D. Ng, Mod. Phys. Lett. A 9, 1975 (1994); P. H. Frampton and D. Ng, Phys. Rev. D 45, 4240 (1992); E. D. Carlson and P. H. Frampton, Phys. Lett. B 283, 123 (1992).

[8] B. Dion, T. Grégoire, D. London, L. Marleau and H. Nadeau, Phys.Rev. D59 (1999).

[9] R. D. Peccei and H. Quinn, Phys. Rev. Lett. 38, 1440 (1977); Phys. Rev. D16, 1791 (1977).

[10] David L. Anderson and Marc Sher, Phys. Rev. D 72, 095014 (2005).

[11] David J. Gross, Jeffrey A. Harvey, Emil Martinec, and Ryan Rohm, Phys. Rev. Lett. 54, 502505 (1985).

[12] Daniel Ng, Phys. Rev. D 49, 48054811 (1994).

[13] V. Pleitez, Phys. Rev. D 61, 057903 (2000).

[14] P. Jain and S. D. Joglekar, Phys. Lett. B 407, 151 (1997).

[15] J. C. Montero, V. Pleitez, M. C. Rodriguez, Phys.Rev. D65 (2002); J. C. Montero, V. Pleitez, M. C. Rodriguez, Phys.Rev. D70 (2004); M. C. Rodriguez, Int.J.Mod.Phys. A21 (2006); P. V. Dong, D. T. Huong, M. C. Rodriguez, H. N. Long, Nucl.Phys.B772:150-174 (2007).

[16] E. Boos et al, Nucl. Instrum. Meth. A534 (2004); A.Pukhov et al, INP MSU report 98-41/542 (arXiv:hep-ph/9908288).

[17] Hoang Ngoc Long and Dang Van Soa, Nucl. Phys. B601:
361-379, (2001).

[18] Paul H. Frampton and Daniel Ng, Phys. Rev. D 45 , 42404245 (1992).

[19] A. Semenov. Nucl.Inst. and Meth. A393 (1997) p. 293.

[20] J. Pumplin et al, JHEP0207:012, (2002).

[21] P. Das, P. Jain, and D. W. McKay, Phys. Rev. D 59, 055011 (1999).

[22] J. G. Duenas, N. Gutierrez, R. Martinez and F. Ochoa, Eur. Phys. J. C 60, 653-659 (2009).

[23] ATLAS Collaboration, "Expected performance of the ATLAS experiment : detector, trigger and physics", CERN-OPEN-2008-020 (2008)

[24] B. Meirose and A.J. Ramalho, Phys. Rev. D73, 075013 (2006); B. Meirose and A.J. Ramalho, J. Phys. G: Nucl. Part. Phys. 36, 095007 (2009).

[25] I. Bertram, G. Landsberg, J. Linnemann, R. Partridge M. Paterno and H.B. Prosper, Fermilab TM2104 (2000); For the "Simple Limit Calculator" see http://www-d0.fnal.gov/Run2Physics/limit_calc/limit_calc.html

[26] CERN Press Office (31 January 2011), "CERN announces LHC to run in 2012".

[27] S. Fartoukh, sLHC Project Report 0049 (http://cdsweb.cern.ch/record/1301180) (2010).

\section{Acknowledgments}

This work has been partially supported by the European Commission within the 7th framework programme (FP7-PEOPLE-2009-IEF, Grant Agreement no. 253339). 